\title{
Contact pressure in misaligned spline couplings
}

\author{
Clément Dupertuis ${ }^{*}$ and Jean-Louis Ligier \\ Haute Ecole d'Ingénierie et de Gestion du Canton de Vaud, HEIG-VD, 1 route de Cheseaux, 1400 Yverdon-les-bains, Suisse
}

Received: 11 November 2019 / Accepted: 9 June 2020

\begin{abstract}
Spline couplings are increasingly being studied because of their growing use, particularly in the field of electric cars. Due to clearances, geometrical obligations and elastic deformation, spline couplings operate mainly in slightly misaligned situations. From these kinds of situations, problems could appear such as vibrations or contact damages. The purpose of this article is to analyse the misalignment of splined couplings and the effects it induces. In order to identify behavioural trends, this work offers analytical approaches for estimating contact pressures in spline connections. In the chosen approach, the simplifying hypotheses necessary for equations are detailed as well as the various analytical modelling. Despites these assumptions, comparisons with pure numerical approaches illustrates the quality of analytical approach adopted in this paper. This method of modelling contact pressure allows the identification of the main mechanical factors to be identified including stiffness, thus deceasing problems due to misalignments.
\end{abstract}

Keywords: Spline couplings / misalignment / contact pressures / modeling / stiffness

\section{Introduction}

Spline coupling is a current component used to transmit a rotating torque from an input (a shaft) to an output (a gear hub). The torque transmission is done thanks to external teeth on the shaft engage with the internal teeth in the hub.

Use areas of splined joint include aerospace, manufacturing, wind power and automotive. In this last domain, current emergence of electrical cars will increase a lot its use and do necessary to get a better understanding of its specific behaviors.

From a mechanical point of view, the main reasons for using such component are its ability to transmit large torque (better mechanical resistance than keyed shaft), to operate at high speed, to accept reverse operating conditions, ease of assembly or disassembly and maintenance. Due to its ability to accommodate some slight misalignment, to accept some axial displacements, this simple and inexpensive joint do not need accurate positioning of the coupled shafts.

Despite its various characteristics, often spline couplings are over dimensioned and remain subject to damages or failures. The most common failure or damage mode observed in spline couplings are wear, fretting-wear [1], fretting-corrosion, fretting fatigue, teeth fatigue [2] and over loading of spline coupling shaft bearing [3]. Some noise and vibration problems have also been encountered in industrial applications [4].

\footnotetext{
* e-mail: clement.dupertuis@heig-vd.ch
}

Optimal design and use of spline couplings are always tricky because it is quite difficult to assess contact behavior and load distribution characteristics [5], particularly when spline teeth have manufacturing errors. Numerical simulations are very hard and require specific codes or development integrating Boundary Element method. First developments with boundary method are twenty years old and have been done by Adey [6].

From this quick overview, it is clear that it is a great challenge to design a coupling joint exactly as is necessary in the early stages of design. To go deeper in detail, we could summarize to four main problems to address to this challenge.

In the first instance, the real operating conditions are never well known, particularly, when the misalignment and geometrical defects of teeth are present. As mentioned by Alfares [7] "we have to live with". Curà and Mura [8] show experimentally the difficulty to correctly evaluate misalignment in true conditions. On the one hand, the importance of the defects is underestimated. On the other hand, the tempering effect of the contact elasticity and of the structure is not properly evaluated.

Secondly, the criteria for ranking the operating conditions of the coupling in relation to the four major damages are not efficient enough to be perfectly predictive. For wear, it is usual to use Archard relationship. But it is well known that this one needs to be adapted and adjusted experimentally as underlined by Fillot [9]. Concerning fretting, and its various forms of damage, there is currently no unanimous criterion. For fretting fatigue, be it the Ruiz criterion or the SWT criterion, experimental calibration is 


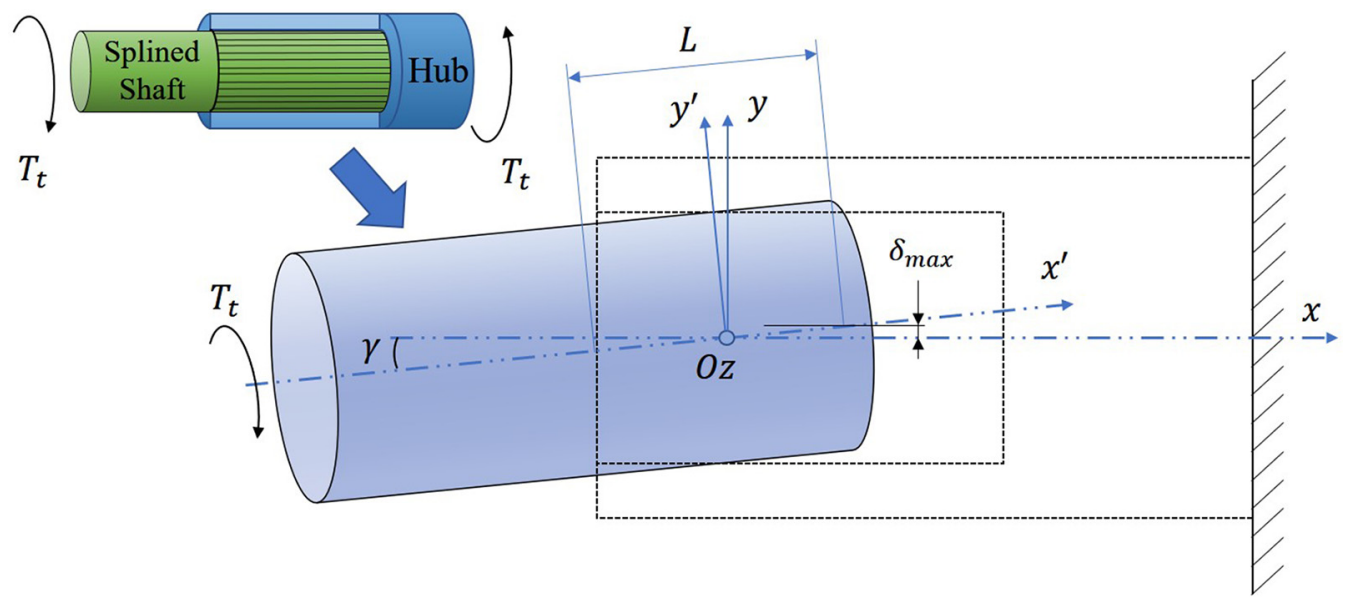

Fig. 1. Simplified representation of misalignment.

necessary to have realistic predictions [10]. For fretting wear this type of comment is confirmed by the large number of publications related to experimental results on this theme $[1,2,11-13]$. For teeth fatigue, except the fact multiaxial criteria needs to be used (Dang Van, Sines, Crossland, etc.), the difficulty lies in having access to the right level of stress.

Thirdly, the calculation of internal stresses in the teeth of this highly hyperstatic contact problem needs specific code. With commercial codes, for instance, it is almost impossible to simulate contact pressures and stresses in the contact areas between tooth with a good level of accuracy when the spline coupling is misaligned. Only a few authors, having developed specific calculation tools, have published on this subject, including Hong and al. [5,14] and Medina and Olver $[15,16]$.

Finally, for dynamic analysis, the global stiffness of the spline coupling is misaligned condition is generally overestimated. Due to its self-alignment capability, the spline coupling in a misaligned situation generates high reaction moments and forces at the shaft supports. These forces, especially on insufficiently preloaded roller bearings, intensify their nonlinear behavior and the generation of vibration over-harmonics $[17,18]$.

Each of the different aspects mentioned above is the subject of research. However, for the engineer who has to design a spline coupling, it is important for him to have a simpler global approach that can eventually constitute his know-how, once this approach has been correlated with the feedback from experience. The present work attempts to be part of this process. For that, we have chosen to use an analytical approach to fully understand the behavior sensitivities regarding the key parameters defining the tooth geometry of the spline coupling. This choice allows us to keep in mind the main mechanical aspects which govern the peaks of contact pressure between teeth. On the other hand, to get a full understanding of this subject interest, it is useful to highlight the fact that there are very few publications of analytical results [7,19-21]. The main and accurate results on misaligned spline coupling were obtained with dedicated simulation tools that are not commercially available $[5,14]$, however, these results could be used as a reference for our approach. Having simple tools allowing evaluating contact pressure peaks, stiffness, slipping, support reactions would be an important help for the designer. The analytical approach makes it possible to provide answers of this type.

By adopting this approach, this implies that several simplifying hypotheses are necessary to treat the subject. These hypotheses will be presented below.

\section{Geometry}

First, it was chosen to model misalignment from a geometric perspective. To this end, some definitions concerning the geometry of splined connections have been laid:

- The material is elastic.

- Misalignment angle is very low $\ll 1^{\circ}$.

- Spline coupling clearance is supposed very small.

- Coulomb friction is taken into consideration.

- The profile of the teeth with developing circle follow the norm NF ISO 4156-1.

- The rotation speed on the splined shaft, written $\omega$ is constant.

In this paper, misalignment will be characterized by:

- Angular misalignment, the acute $\gamma$ angle between the axes of rotation of the female and male parts.

- The parallel misalignment of $\delta_{p}$ (offset), that is, the distance between the shaft axis and the female axis at the centre of the common part of the male and female teeth.

Thus, from the angle $\gamma$ and the active length of the tooth $L$ (Fig. 1), it is possible to express the maximum height $\delta_{\max }$ of the toothing end in the case of a misalignment. For any gamma angle misalignment and a parallel $\delta_{p}$ offset on the y-axis, the maximum local misalignment will be:

$$
\delta_{\max }=\delta_{p}+\frac{L}{2} \cdot \sin \gamma
$$

\subsection{Geometric interferences}

In this section we construct the modelling of the geometric interference between the teeth of the male and female 


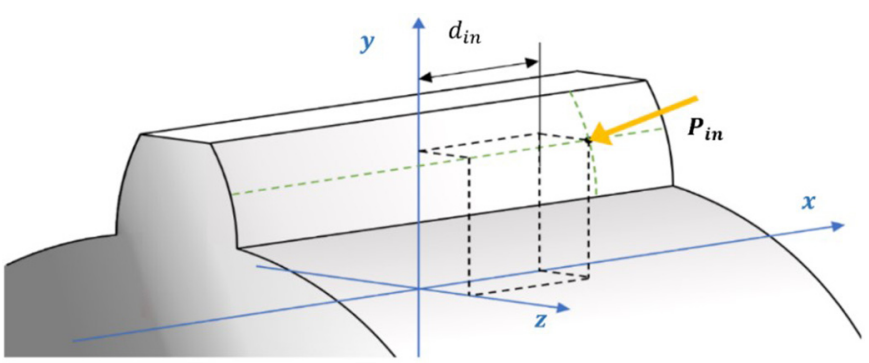

Fig. 2. Force application distances.

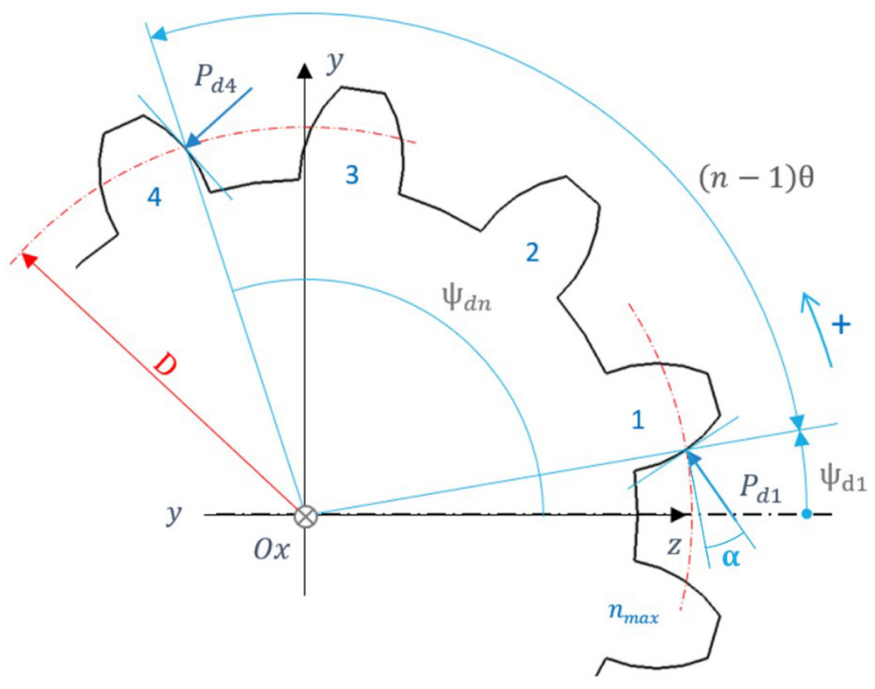

Fig 3. Geometrical variables and loads on the shaft.

parts. These interferences are directly related to contact pressures between teeth.

Thus, as a first step, we have chosen to assume that there are internal loadings on all teeth. These efforts are unknown, but we can express the produced resulting torsor (reduced torsor at the centre of the splined bond). Forces and moments acting on the toothing in the presence of misalignment are considered. Shear loadings result from the sliding rubbing between the teeth. Each contact loading has been assumed to act on the primary diameter for the radial direction and are identified by the variable $d_{i n}$ for their axial positioning. As for the direction of these efforts in the plan $(y, z)$ and their position, this is defined in Figures 2 and 3 for a spline coupling having $n_{\max }$ teeth.

Relationships for moment and force resultant at the point $O$ can be expressed with reference to the axis $x, y, z$. Therefore, we consider the angle of offset between the teeth $\theta=2 \pi / n_{\max }$ and $\mu$ the kinematic friction coefficient.

With the representation given in Figure 4, we obtain the following expression for the torsor:

$$
\begin{gathered}
T=\sum_{n=1}^{n_{\max }}\left(P_{d n}-P_{g n}\right) \cdot \frac{D}{2} \cos (\alpha) \\
N_{x}=\mu \sum_{n=1}^{n_{\max }}\left(P_{d n} \cdot \operatorname{sgn}\left(\cos \left(\psi_{d n}\right)\right)+P_{g n} \cdot \operatorname{sgn}\left(\cos \left(\psi_{g n}\right)\right)\right)
\end{gathered}
$$

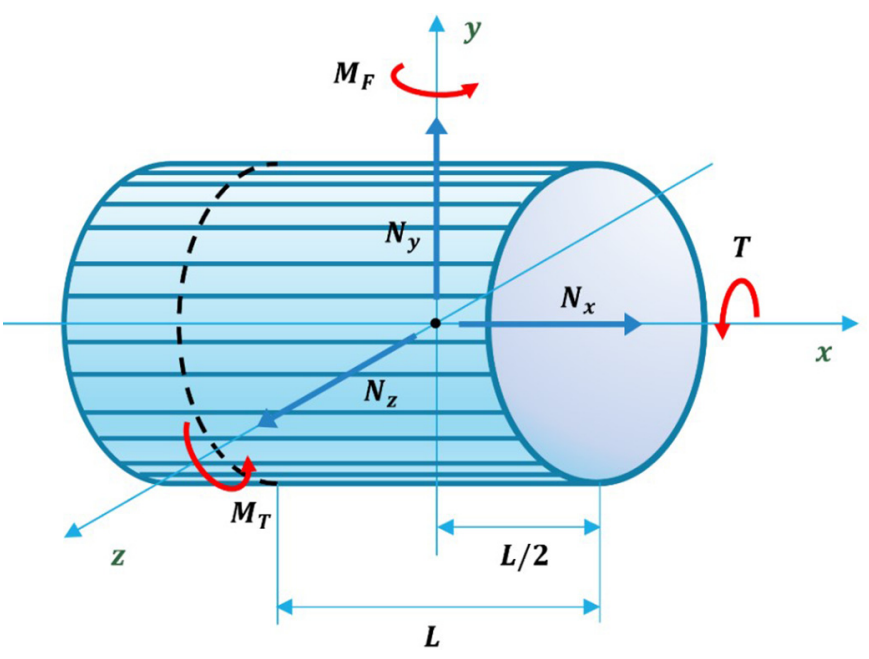

Fig. 4. Forces and torques on the splined shaft.

$$
\begin{gathered}
M_{F}=M_{f r, y}+M_{p, y} \\
N_{y}=\sum_{n=1}^{n_{\max }}\left(P_{d n} \cdot \cos \left(\psi_{d n}+\alpha\right)-P_{g n} \cdot \cos \left(\psi_{g n}-\alpha\right)\right) \\
M_{T}=M_{f r, z}+M_{p, z} \\
N_{z}=\sum_{n=1}^{n_{\max }}\left(-P_{d n} \cdot \sin \left(\psi_{d n}+\alpha\right)+P_{g n} \cdot \sin \left(\psi_{g n}-\alpha\right)\right) .
\end{gathered}
$$

The moments of friction $M_{f r, y}$ et $M_{f r, z}$ are expressed as:

$$
\begin{aligned}
M_{f r, y}= & -\mu \sum_{n=1}^{n_{\max }}\left(P_{\mathrm{dn}} \cos \left(\psi_{d n}\right) \cdot \operatorname{sgn}\left(\cos \left(\psi_{d n}\right)\right)\right. \\
& \left.+P_{g n} \cos \left(\psi_{g n}\right) \cdot \operatorname{sgn}\left(\cos \left(\psi_{g n}\right)\right)\right) \cdot \frac{D}{2} \\
M_{f r, z}= & -\mu \sum_{n=1}^{n_{\max }}\left(P_{d n} \sin \left(\psi_{d n}\right) \cdot \operatorname{sgn}\left(\cos \left(\psi_{d n}\right)\right)\right. \\
& \left.+P_{g n} \sin \left(\psi_{g n}\right) \cdot \operatorname{sgn}\left(\cos \left(\psi_{g n}\right)\right)\right) \cdot \frac{D}{2} .
\end{aligned}
$$

And the moments of support $M_{p, y}$ and $M_{p, z}$ as:

$$
M_{p, y}=\sum_{n=1}^{n_{\max }}\left(P_{d n} \cdot d_{d n} \cdot \sin \left(\psi_{d n}+\alpha\right)-P_{g n} \cdot d_{g n} \cdot \sin \left(\psi_{g n}-\alpha\right)\right)
$$

$$
M_{p, z}=\sum_{n=1}^{n_{\max }}\left(P_{d n} \cdot d_{d n} \cdot \cos \left(\psi_{d n}+\alpha\right)-P_{g n} \cdot d_{g n} \cdot \cos \left(\psi_{g n}-\alpha\right)\right) .
$$

In this contact problem, it can be noted that this is a highly hyperstatic problem which requires consideration of 


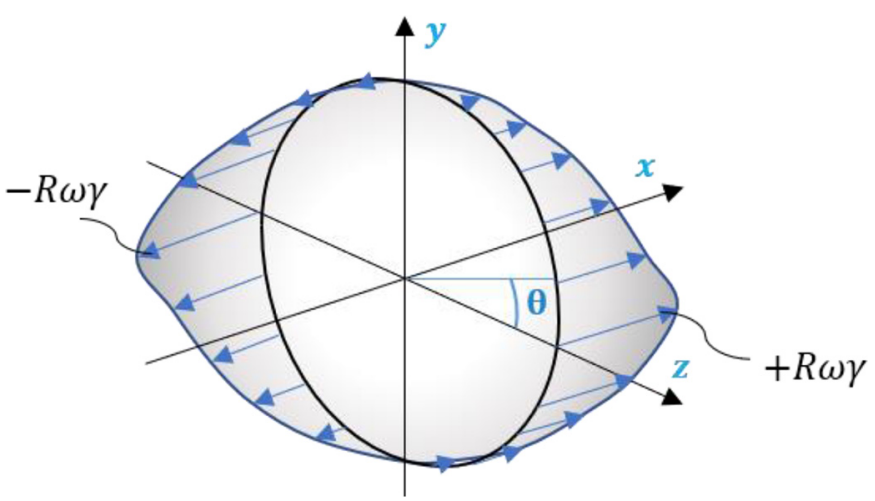

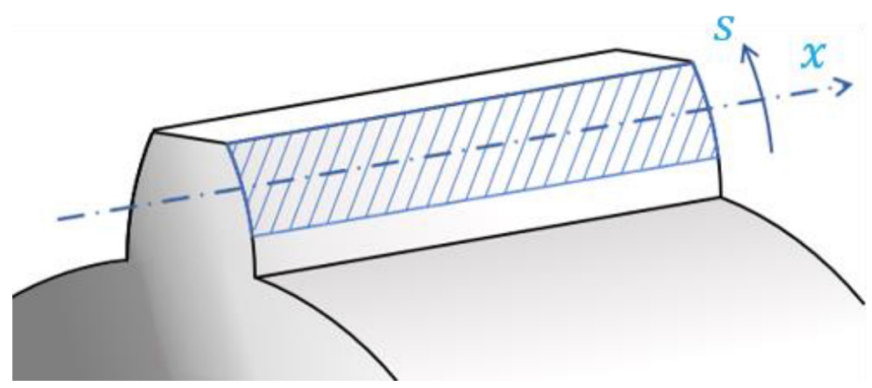

Fig. 6. Contact pressure application area.

Fig. 5. 3D Representation of the slippage profile.

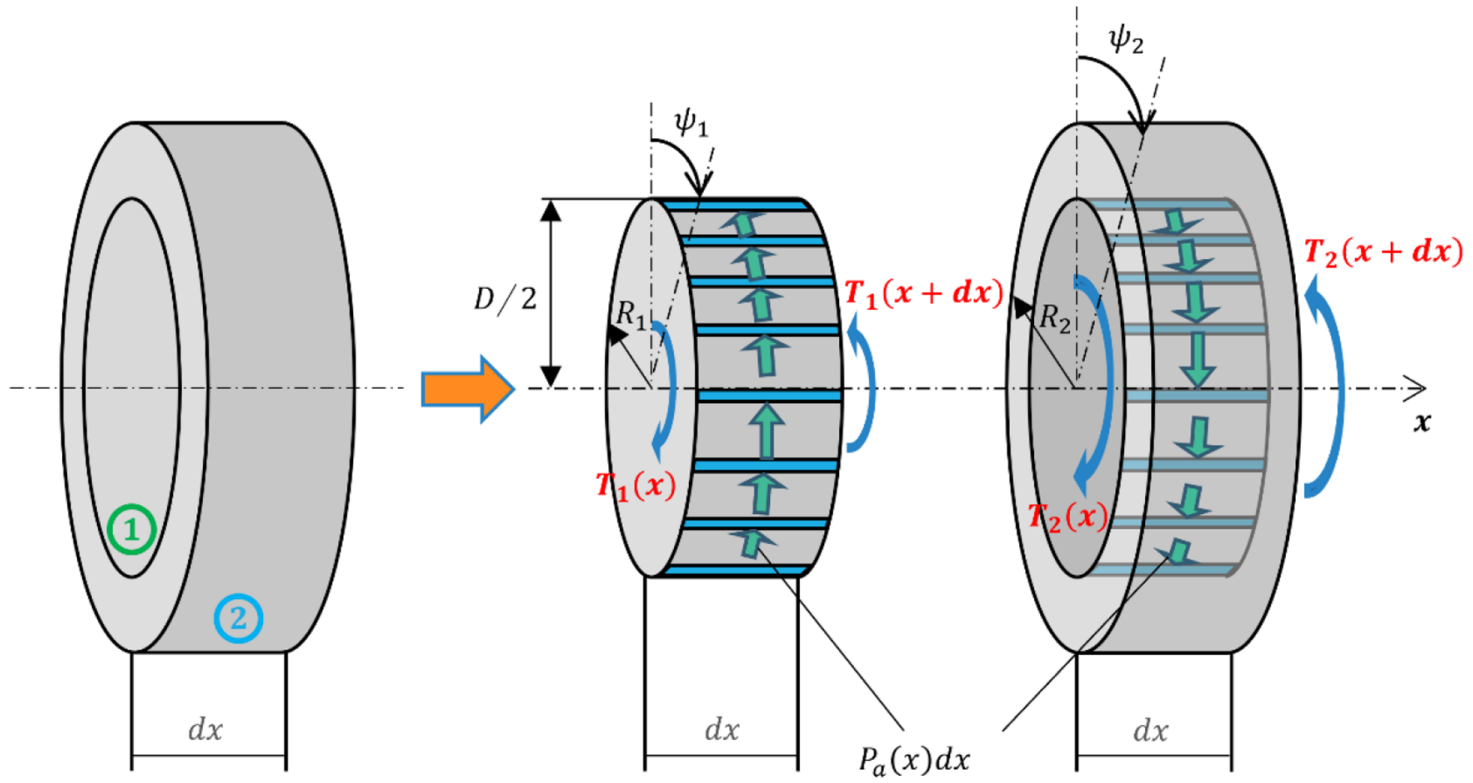

Fig. 7. Representation of an infinitesimal portion of the splined connection.

the deformation aspects of the teeth through stiffness. Stiffness modelling is given below.

For a $C$ point contact, common between the two misaligned parts, it is possible to express the sliding speed in the shaft coordinates in the following form (second order of $\gamma$ has been neglected):

$$
\Delta \overrightarrow{v_{\mathrm{Cr}}} \simeq\left[\begin{array}{c}
R \omega \cdot \cos \theta \cdot \gamma \\
0 \\
0
\end{array}\right] .
$$

By plotting this relative speed on a circular graph, we obtain the speed profile represented by Figure 5. This representation illustrates the friction acting on the shaft as friction forces will have the same orientation than the slippage.

\subsection{Contact pressure field}

From splined gear literature [22] it seems more appropriate to look successively at how the contact pressures vary axially (according to $x$ ), then along the profile (according to the curvilinear abscissa $s$ ), as is presented in Figure 6 .

\subsubsection{Evolution of the axial lineic force}

To analyse the axial evolution, we have used a common modelling from literature [23-25]. Thus, the contact lineic force in the axial direction can be expressed as the torque variation over an infinitesimal interval along the $x$ axis.

Therefore, on the assumption that all the teeth of the coupling are in perfect contact and that the forces can be linearly summed, we can investigate the equilibrium of a slice of thickness dx according to the diagram in Figure 7.

From the static torsional equilibrium, the following equation system is obtained, where GI is the torsional stiffness and $\psi_{1}, \psi_{2}$ the rotational angle of each parts of the coupling:

$$
\left\{\begin{array}{l}
G_{1} I_{1} \frac{d^{2} \psi_{1}}{d x^{2}}-K \cdot\left(\psi_{1}-\psi_{2}\right)=0 \\
G_{2} I_{2} \frac{d^{2} \psi_{2}}{d x^{2}}+K \cdot\left(\psi_{1}-\psi_{2}\right)=0
\end{array}\right.
$$


The $K$ notation is used to define the torsional stiffness of teeth contact per unit width. This stiffness is multiple of the resulting stiffness for two antagonistic teeth of a shaft/ hub torque in contact.

By defining the factors $K_{i}=K / G_{i} I_{i}$ and $K_{T}=\sqrt{K_{1}+K_{2}}$, the solution of this system can be simplified. The boundary conditions use for solving the differential equations are:

- For $x=0: T_{2}=0$ et $T_{1}=T_{t}$.

- For $x=L: T_{1}=0$ et $T_{2}=T_{t}$.

- For $0 \leq x \leq L: T_{t}=T_{1}(x)+T_{2}(x)$.

After integration, angle and torque solutions, for the shaft and the hub respectively, have the expressions:

\section{See equations (14) and (15) below.}

Due to the hypothesis of perfect teeth contact we can analyse the loading on each tooth.

With an axial lineic contact force $P_{a}(x) d x$ applied to each of the teeth of the fluted shaft, as represented in Figure 7, the torsional balance of these two slices allows the expression of axial lineic force:

$$
P_{a}(x)=\frac{1}{n_{\max } \frac{D}{2} \cos (\alpha)} \frac{d T_{1}(x)}{d x}=-\frac{1}{n_{\max } \frac{D}{2} \cos (\alpha)} \frac{d T_{2}(x)}{d x} .
$$

So, for each shaft teeth, we get the following contact force:

$$
\begin{aligned}
P_{a}(x)= & \frac{1}{n_{\max } \frac{D}{2} \cos (\alpha)} \frac{K_{T} K_{1} T_{t}}{K_{2}+K_{1}} \\
& \times\left(-\frac{\frac{K_{2}}{K_{1}}+\cosh \left(K_{T} L\right)}{\sinh \left(K_{T} L\right)} \cosh \left(K_{T} x\right)+\sinh \left(K_{T} x\right)\right)
\end{aligned}
$$

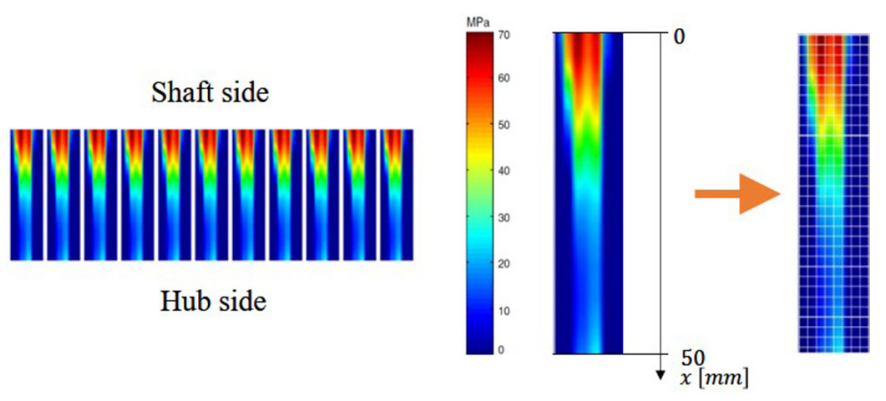

Fig. 8. Pressure field on the toothing with a Torque applied $T=2200 \mathrm{Nm}$. [26].

The full development can be found on the literature

\subsubsection{Validation of the modelling}

To confirm the pertinence of this approach we have compared our results with results from the literature. One of the more accurate result has been produced by Hong [5]. The contact pressure field on shaft tooth extracted from this work is presented in Figure 8.

From these results, with an integration on the curvilinear abscissa, we can get the lineic contact force along the axis $x$. Taking in consideration the characteristics of the Hong modelling in our own modelling and considering tooth stiffness taken at $1.04 \times 10^{9} \mathrm{~N} / \mathrm{rad}$, lineic force from the two approaches are very close (Fig. 9).

\subsubsection{Contact pressure on the curvilinear abscissa}

As several authors have considered [22] a punch model to describe the contact pressure variation along the curvilinear axis of the teeth, we shall adopt the same approach. For each tooth in contact, deformation results from a translation

$$
\begin{aligned}
& \left\{\begin{array}{c}
\psi_{1}=\frac{T_{T}}{G_{1} I_{1}\left(1+\frac{K_{1}}{K_{2}}\right)} x+\frac{-T_{T}\left(\frac{K_{2}}{K_{1}}+\cosh \left(K_{T} L\right)\right) \cosh \left(K_{T} x\right)}{\sinh \left(K_{T} L\right) \cdot G_{1} I_{1} K_{T}\left(1+\frac{K_{2}}{K_{1}}\right)}+\frac{T_{T} \sinh \left(K_{T} x\right)}{G_{1} I_{1} K_{T}\left(1+\frac{K_{2}}{K_{1}}\right)} \\
\psi_{2}=\frac{T_{T}}{G_{1} I_{1}\left(1+\frac{K_{1}}{K_{2}}\right)}\left(x+\frac{\left(\frac{K_{2}}{K_{1}}+\cosh \left(K_{T} L\right)\right) \cosh \left(K_{T} x\right)}{\sinh \left(K_{T} L\right) K_{T}}-\frac{T_{T} \sinh \left(K_{T} x\right)}{K_{T}}\right)
\end{array}\right. \\
& \left\{\begin{array}{l}
T_{1}=\frac{T_{t}}{1+\frac{K_{1}}{K_{2}}}\left(1+\frac{K_{1}}{K_{2}}\left(-\frac{\frac{K_{2}}{K_{1}}+\cosh \left(K_{T} L\right)}{\sinh \left(K_{T} L\right)} \sinh \left(K_{T} x\right)+\cosh \left(K_{T} x\right)\right)\right) \\
T_{2}=\frac{T_{t}}{1+\frac{K_{2}}{K_{1}}}\left(1+\frac{\frac{K_{2}}{K_{1}}+\cosh \left(K_{T} L\right)}{\sinh \left(K_{T} L\right)} \sinh \left(K_{T} x\right)-\cosh \left(K_{T} x\right)\right)
\end{array} .\right.
\end{aligned}
$$




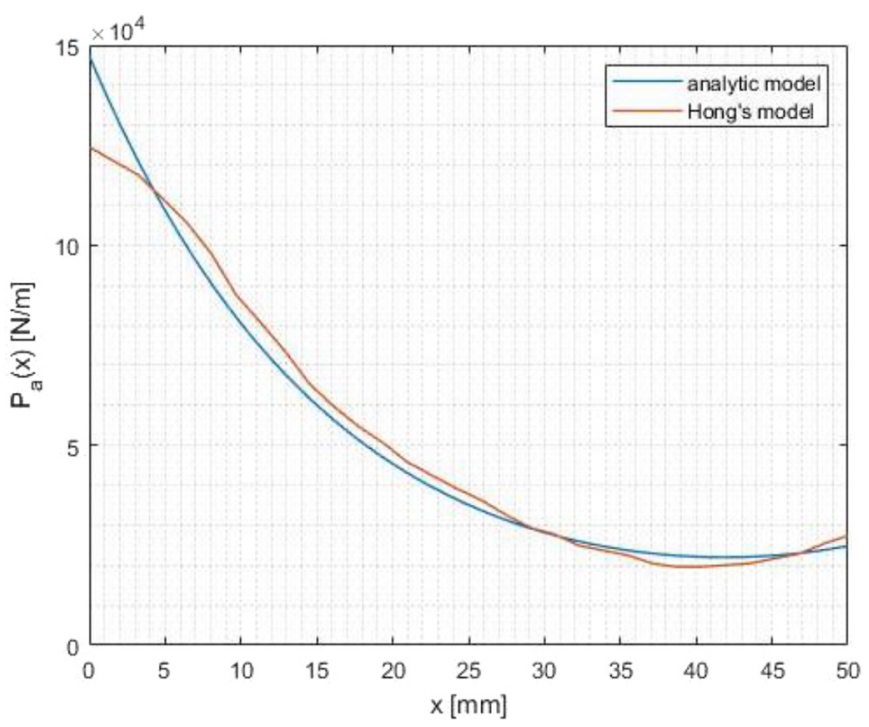

Fig. 9. Comparison of the two model.

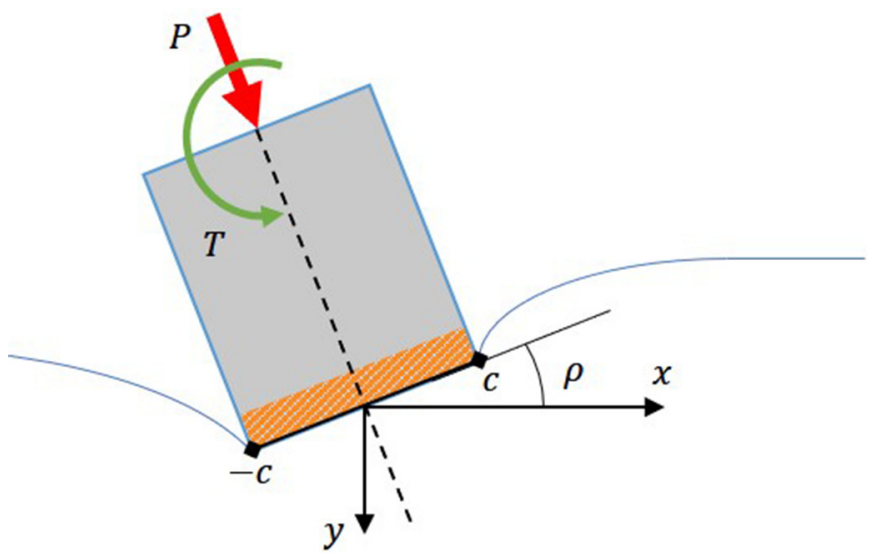

Fig. 10. Punch illustration.

and a rotation (Fig. 11), then it seems adequate to consider the contact pressure given by Sackfield [27] for a punch subject to normal load and a moment (Fig. 10).

$$
p(x)=\frac{1}{\sqrt{c^{2}-x^{2}}}\left(\frac{P}{\pi}+\frac{\rho}{A} x\right) .
$$

With the factor $A=\frac{\chi+1}{4 G}\left(\mathrm{~N} / \mathrm{m}^{2}\right) \cdot \chi$ and $G$ come from the material and are respectively the Kolosov constant $(\chi=3-4 v)$ and the shear modulus $G$.

Since this model gives an infinite contact pressure at the ends of the contact area, we introduce some corrections proposed by Barrot [22] with $a^{\prime}>a$ and $P_{i n}>P$ to get finite realistic value of pressure. The expression of the pression

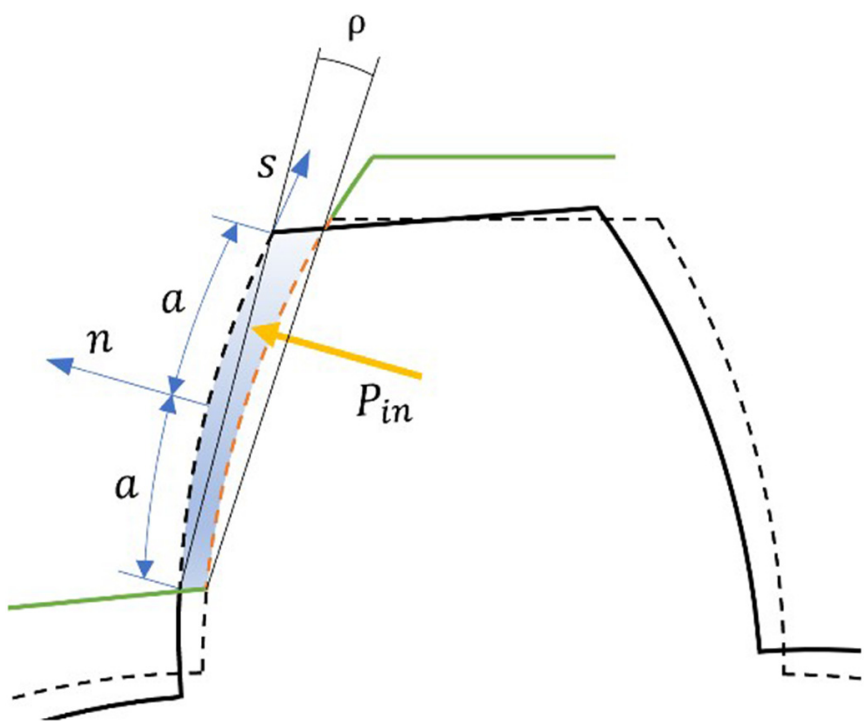

Fig. 11. Representation on our model.

then becomes:

$$
P_{c}(s)=\frac{1}{\sqrt{a^{\prime 2}-s^{2}}}\left(\frac{P_{i n}}{\pi}+\frac{\rho}{A} s\right) .
$$

In summary, a soon as the lineic contact force is determined, maximum pressure can be extrapolated from the contact expression of the corrected punch model.

\subsubsection{Teeth stiffness}

Because of the multiple hyperstatic contacts, we described the teeth stiffness per unit of length [25]. In this approach we regard the stiffness as independent of the position $x$ on the teeth. Thus, we consider that this stiffness can be split in three main contributions with their mathematic expressions.

First, the embedment stiffness can be described by the following displacement due to a force $F$ applied on the primary radius [28]:

$$
u_{e n c}=\frac{F}{L} \frac{1-v^{2}}{E} \cos ^{2} \alpha
$$

$$
\left(10.92 \int_{0}^{h} \frac{(h-y)^{2}}{b^{3}(y)} d y+3.1\left(1+0.294 \tan ^{2} \alpha\right) \int_{0}^{h} \frac{d y}{b(y)}\right)
$$

Second, the displacement due to contact stiffness can be expressed with Hertz relationships [28]:

$$
u_{c o}=2 \frac{F}{L} \frac{1-v^{2}}{\pi E}\left(\ln \frac{a_{1}}{A}-\frac{v}{2(1-v)}\right)
$$

With $A=\sqrt{8 \frac{F}{L} a_{0} \frac{1-v^{2}}{\pi E}}$ the semi-width of the Hertzian contact. 
The different characteristics used in these formulas are relatives to Figure 12 .

Last, shear and flexion stiffness can be calculated with the following relation resulting directly from strength material theory and considering teeth section as trapezoidal:

$$
\begin{aligned}
u_{r d m}= & \frac{6 F H}{E L b_{0}^{3}}\left(-h(h+2 H)+H^{2} \cdot \ln \left(\frac{H}{H-h}\right)\right) \\
& +\frac{F H}{k_{s r} G L b_{0}} \ln \left(\frac{H}{H-h}\right) .
\end{aligned}
$$

With $k_{s r}$ the reduced cross-sectional coefficient in shear.

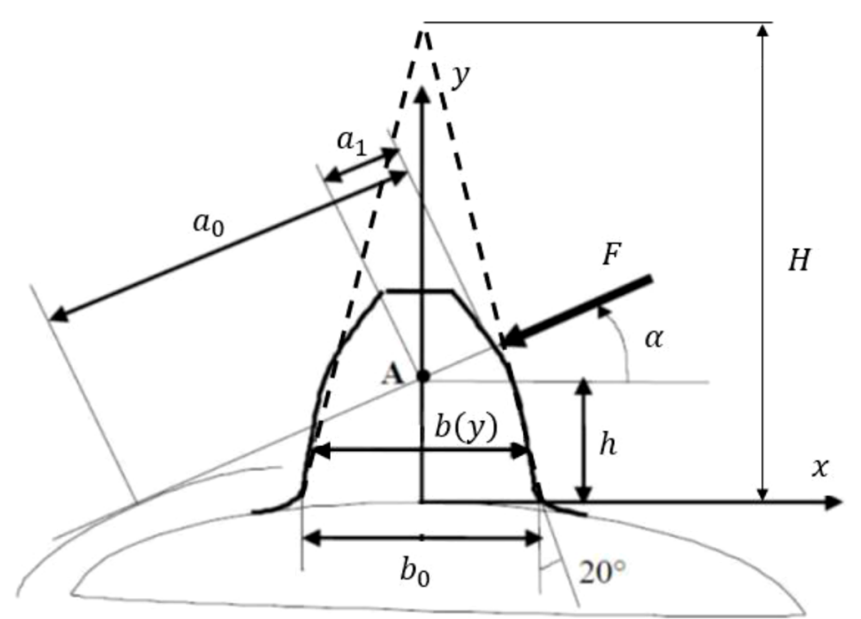

Fig. 12. Teeth characteristics [28].
From this result, we can determine the stiffness $K_{t}$ :

$$
K_{t}=\frac{n F R_{p}^{2}}{L\left(u_{e n c}+u_{c o}+u_{r d m}\right)} .
$$

Thus, with the geometrical characteristics from Hong model, the stiffness is:

$$
K_{t}=0.99 \times 10^{9} \mathrm{~N} / \mathrm{rad} .
$$

This value obtained from elasticity consideration is in perfect agreement with the previous one, $\boldsymbol{K}=\mathbf{1 . 0 4} \times \mathbf{1 0}^{\mathbf{9}} \mathrm{N} / \mathbf{r a d}$, obtained by curve fitting.

\subsubsection{Misalignment modelling}

According to the $\psi$ angle of the tooth (angular position in the plane $(y, z)$ ) and according to the face that is in contact (right or left), the contact area is on the front or back of the tooth. For modelling, it is necessary to establish a relation between the $\gamma$ angular misalignment angle and the normal misalignment of each tooth $n$. For a coordinate point $(x, \theta)$ located on the fictitious primitive cylinder of the shaft, the misalignment according to the tangential direction is expressed as follows:

$$
\delta_{n}(x)=\gamma \cdot x \cdot \cos \theta
$$

We then consider the profiles of the spline sections in Figure 13. On it, the profile of the shaft perfectly aligned with hub profile is represented with the green line. The misaligned shaft appears in blue line, while the profile of the misaligned shaft subjected to torque is represented in red line. From this representation, we can express the condition

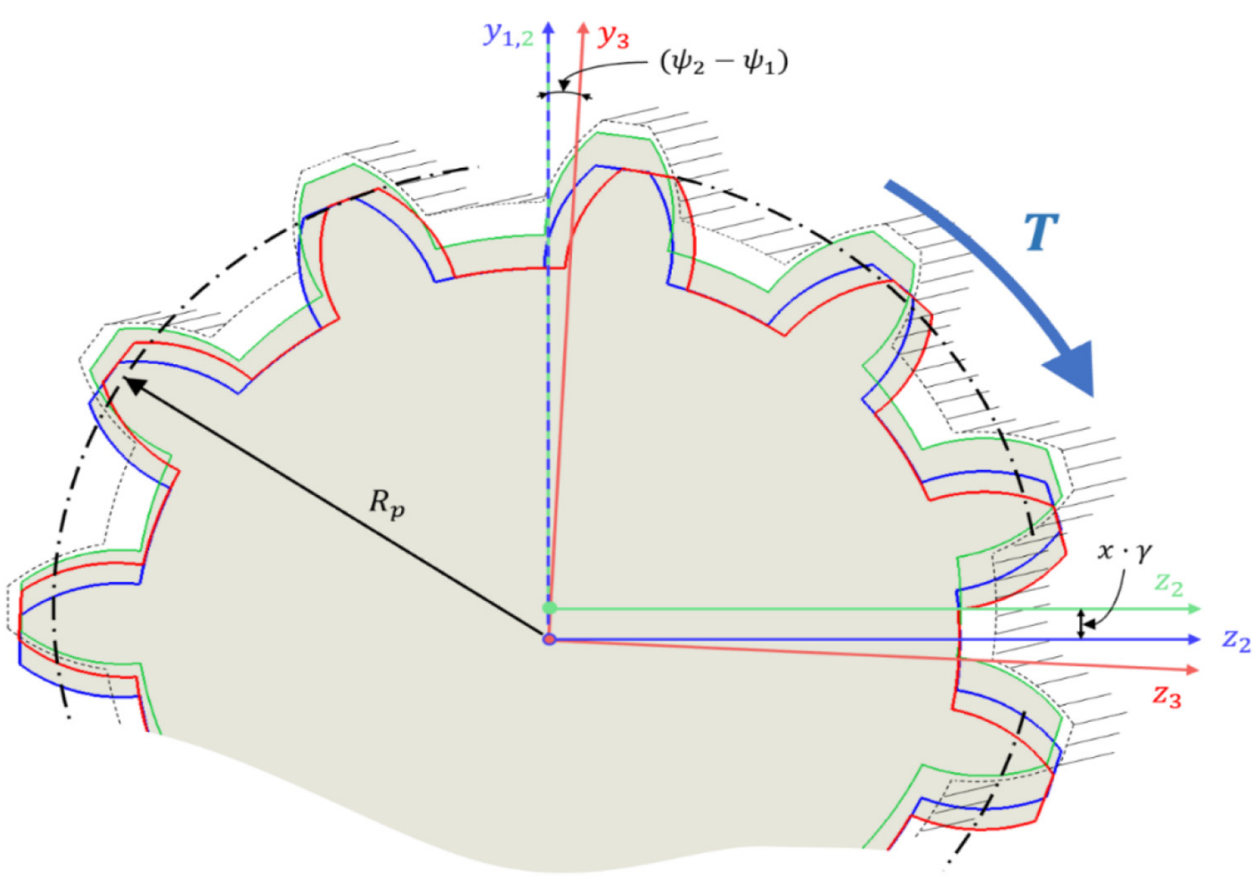

Fig. 13. Misalignment and torque situations. 

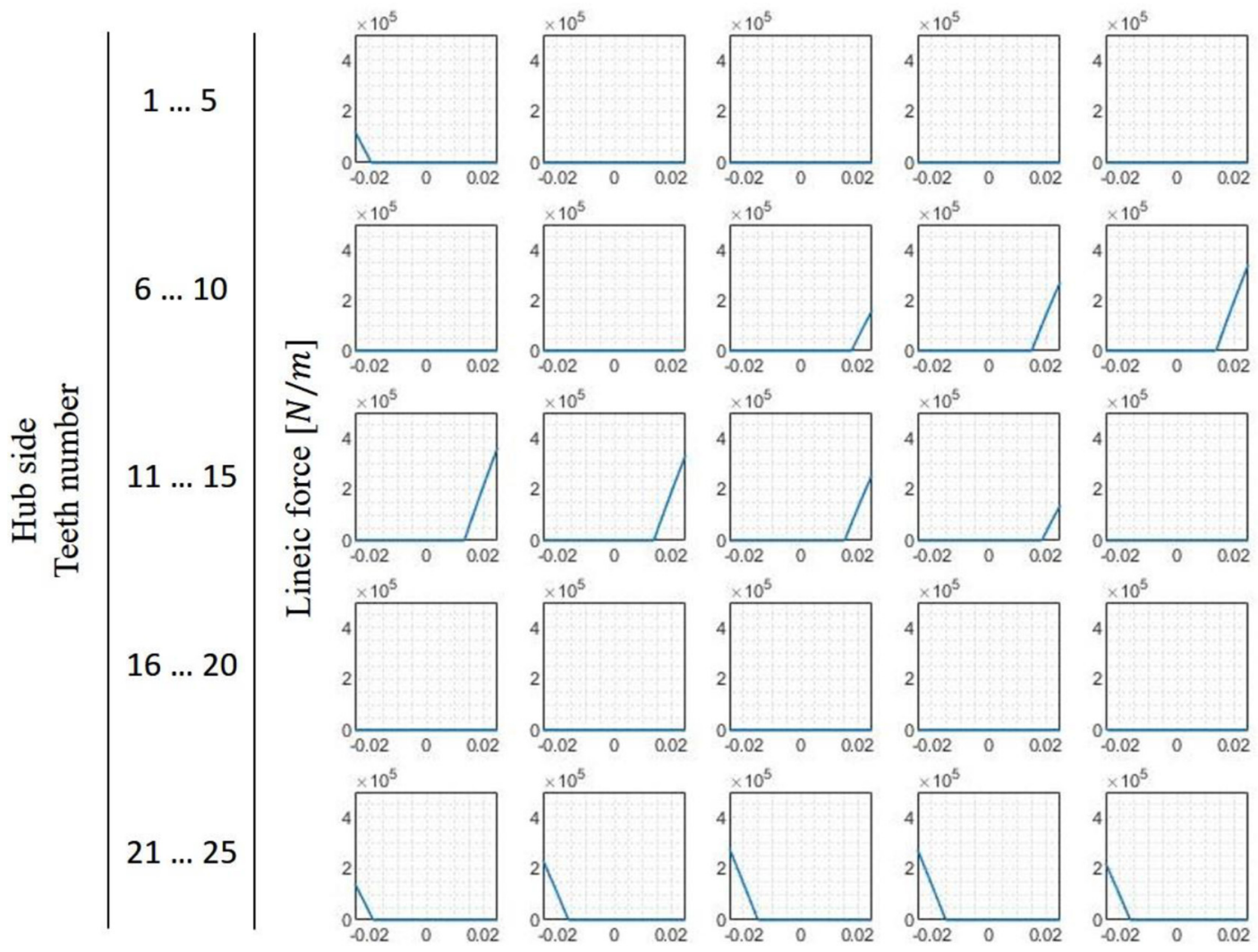

क्ष
है
त
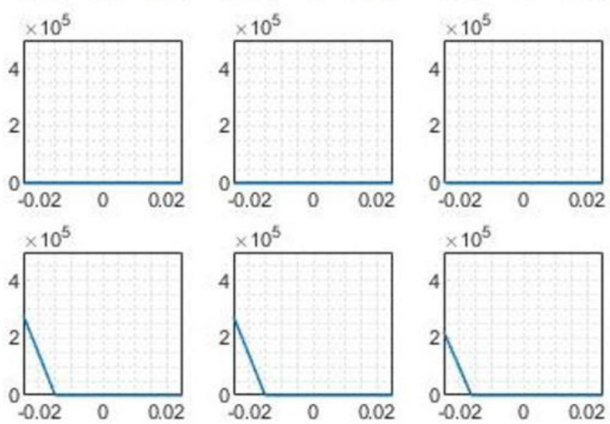

Position $[m]$

Fig. 14. Axial lineic forces.

of contact between teeth by the following relation:

$$
\begin{gathered}
\text { Right side }: \\
\delta_{n d}(x)-R_{p}\left(\psi_{1}(x)-\psi_{2}(x)\right) \\
>J / 2 \Leftrightarrow C_{n d}(x)=\text { TRUE }
\end{gathered}
$$

$$
\text { Left side }:-\delta_{n g}(x)+R_{p}\left(\psi_{1}(x)-\psi_{2}(x)\right)
$$$$
>J / 2 \Leftrightarrow C_{n g}(x)=\text { TRUE. }
$$

And the number of teeth in contact as:

$$
\begin{aligned}
& n_{c d}(x)=\sum_{n=1}^{n_{\max }} C_{n d}(x)=\text { TRUE } \\
& n_{c g}(x)=\sum_{n=1}^{n_{\max }} C_{n g}(x)=\text { TRUE }
\end{aligned}
$$

This allows to define the depth of the interference on the faces of the $n$ teeth in contact, called int ${ }_{n d}$ and int ${ }_{n g}$, by the relation:

$$
\begin{gathered}
\operatorname{int}_{n d}(x)=\max \left(0, \delta_{n d}(x)-R_{p}\left(\psi_{1}(x)-\psi_{2}(x)\right)-J / 2\right) \\
\operatorname{int}_{n g}(x)=\max \left(0,-\delta_{n g}(x)+R_{p}\left(\psi_{1}(x)-\psi_{2}(x)\right)-J / 2\right)
\end{gathered}
$$

\subsubsection{Evolution of the axial lineic force}

Thus, for a left or right contact of an index tooth $n$ whose one side touches its counterpart, the contact linear force developed according to the normal left or right contact direction, for a portion of tooth $d x$ can be expressed as:

$$
d F_{n d}=\frac{\delta_{n d}(x)-R_{p}\left(\psi_{1}(x)-\psi_{2}(x)\right)-J / 2}{R_{p} \cdot \cos \alpha} \cdot K_{e l} \cdot d x
$$

$$
d F_{n g}=\frac{-\delta_{n g}(x)+R_{p}\left(\psi_{1}(x)-\psi_{2}(x)\right)-J / 2}{R_{p} \cdot \cos \alpha} \cdot K_{e l} \cdot d x .
$$

Using Figure 7, it is possible to express the torsional balance, which enables the relationship between the sum of the linear forces and the torque derivative to be obtained as follows:

$$
\begin{aligned}
\sum_{n=N_{C 1}}^{N_{c \max }}\left(d F_{n d}(x)-d F_{n g}(x)\right) & =\frac{1}{\frac{D}{2} \cos (\alpha)} \frac{d T_{1}(x)}{d x} \\
& =-\frac{1}{\frac{D}{2} \cos (\alpha)} \frac{d T_{2}(x)}{d x}
\end{aligned}
$$




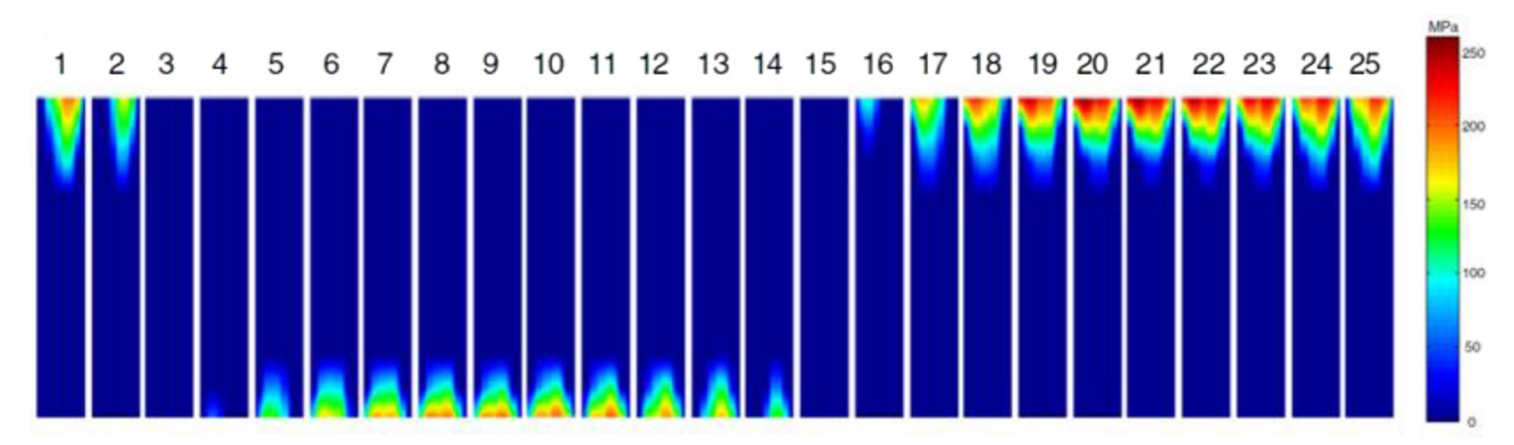

Fig. 15. Hong results.

Another way to express the balance lend to the following equations system:

$$
\left\{\begin{array}{l}
G_{1} I_{1} \frac{d^{2} \psi_{1}}{d x^{2}}-R_{p} \cdot\left(\sum_{n=1}^{n_{c d}(x)} d F_{n d}-\sum_{n=1}^{n_{c g}(x)} d F_{n g}\right)=0 \\
G_{2} I_{2} \frac{d^{2} \psi_{2}}{d x^{2}}+R_{p} \cdot\left(\sum_{n=1}^{n_{c d}(x)} d F_{n d}-\sum_{n=1}^{n_{c g}(x)} d F_{n g}\right)=0
\end{array}\right.
$$

Considering the complexity of this model, we use a numerical solver, and did some manual iteration according to the number of unknown variable, to get a result with the different expressions and the Hong's model characteristics [5]. It corresponds to introduce the following parameters on our model:

\begin{tabular}{llll}
\hline$T=2200 N$ & $\left.\left(\psi_{1}-\psi_{2}\right)\right|_{x=0}=0.02^{\circ}$ & $\gamma=0.12^{\circ}$ & $\alpha=30^{\circ}$ \\
\hline$n=25 \operatorname{dents}$ & $I_{1}=3.62 \times 10^{-6} \mathrm{~m}^{4}$ & $I_{2}=4.57 \times 10^{-5} \mathrm{~m}^{4}$ & $R_{p}=40 \mathrm{~mm}$ \\
$L=50 \mathrm{~mm}$ & $K=1.04 \times 10^{9} \mathrm{~N} / \mathrm{rad}$ & $G_{1}=G_{2}=81 \mathrm{GPa}$ & $J=95 \mu \mathrm{m}$ \\
\hline
\end{tabular}

Thus, we find the following profiles of lineic forces on the 25 teeth (Fig. 14) where the lineic contact force is plotted along the vertical axis with respect to the horizontal $x$ axis.

From these lineic forces results, we calculate the corresponding torsors with the relationships (2)-(3)(4) $-(5)-(6)-(7)$ and find respectively $T=2197 \mathrm{Nm}$, $M_{F}=108 \mathrm{Nm}$ and $M_{T}=-561 \mathrm{Nm}$ for the torques and $N_{x}=12.7 \mathrm{kN}, N_{y}=-50.5 \mathrm{kN}$ and $N_{z}=-38.3 \mathrm{kN}$ for the forces. This means that to get an angular misalignment, the hub and the shaft must withstand extra moment and load. In the present case, with a torque of $2200 \mathrm{Nm}$ and a misalignment of $0.12^{\circ}$, an extra moment of $560 \mathrm{Nm}$ is generated ( $\simeq 25 \%$ of the torque). By knowing the nominal stiffness of the bearings supporting the shaft and the hub it is possible to estimate what will be the true misalignment.

In comparison, the results of Hong have the following form (Fig. 15)

A detail of the comparison of our model with Hong's results is presented in Figure 16 for the maximum force peaks.
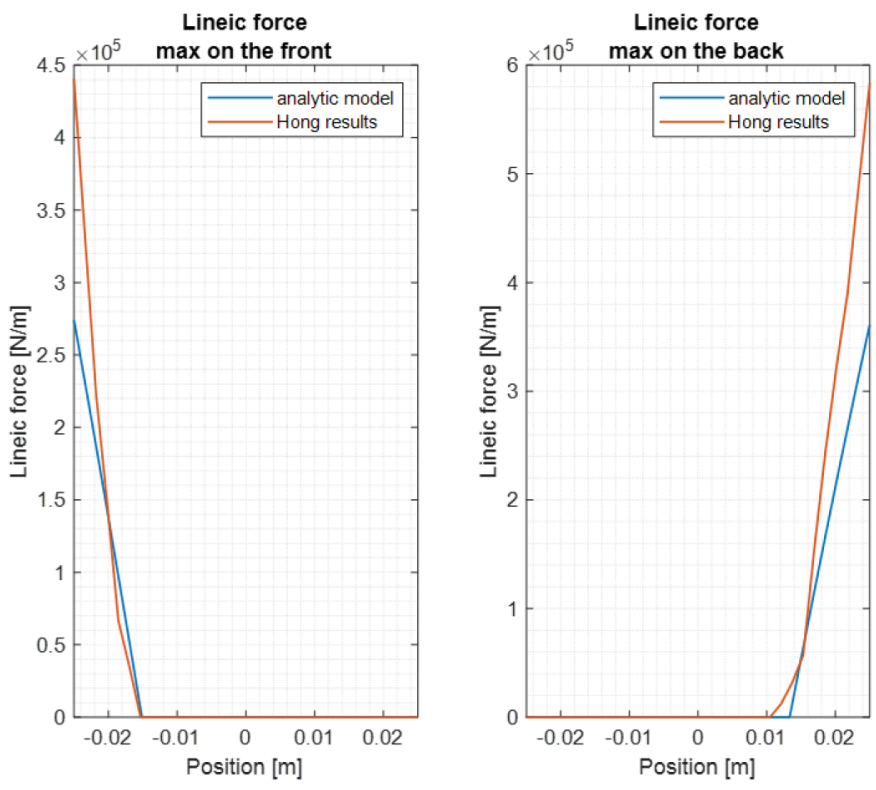

Fig. 16. Comparison between Hong peak results and our model.

Because of the importance of moments and reaction forces, and in order to confirm the benefits of our approach, a comparison was made with Hong's reference results using a $0.12^{\circ}$ misalignment angle. We choose the largest angle of misalignment because it induces a broader dispersion on our/the results. After all computations, the comparison displays a good correlation, as illustrated in Figure 17.

\section{Conclusion}

For the design engineer, defining an optimal spline coupling in terms of dimensions remains a real challenge because there are no commercial codes allowing him to simulate the behavior of this joint in a misaligned situation. However, any spline coupling works more or less in a misaligned situation and it is in this type of situation that problems can arise such as the destruction of the component. In this work modelling the contact between the teeth of the shaft and the hub of the spline coupling, we have deliberately focused on an analytical approach. The main target was to provide answers that are easy for a design engineer to integrate in his design process. With this analytical 


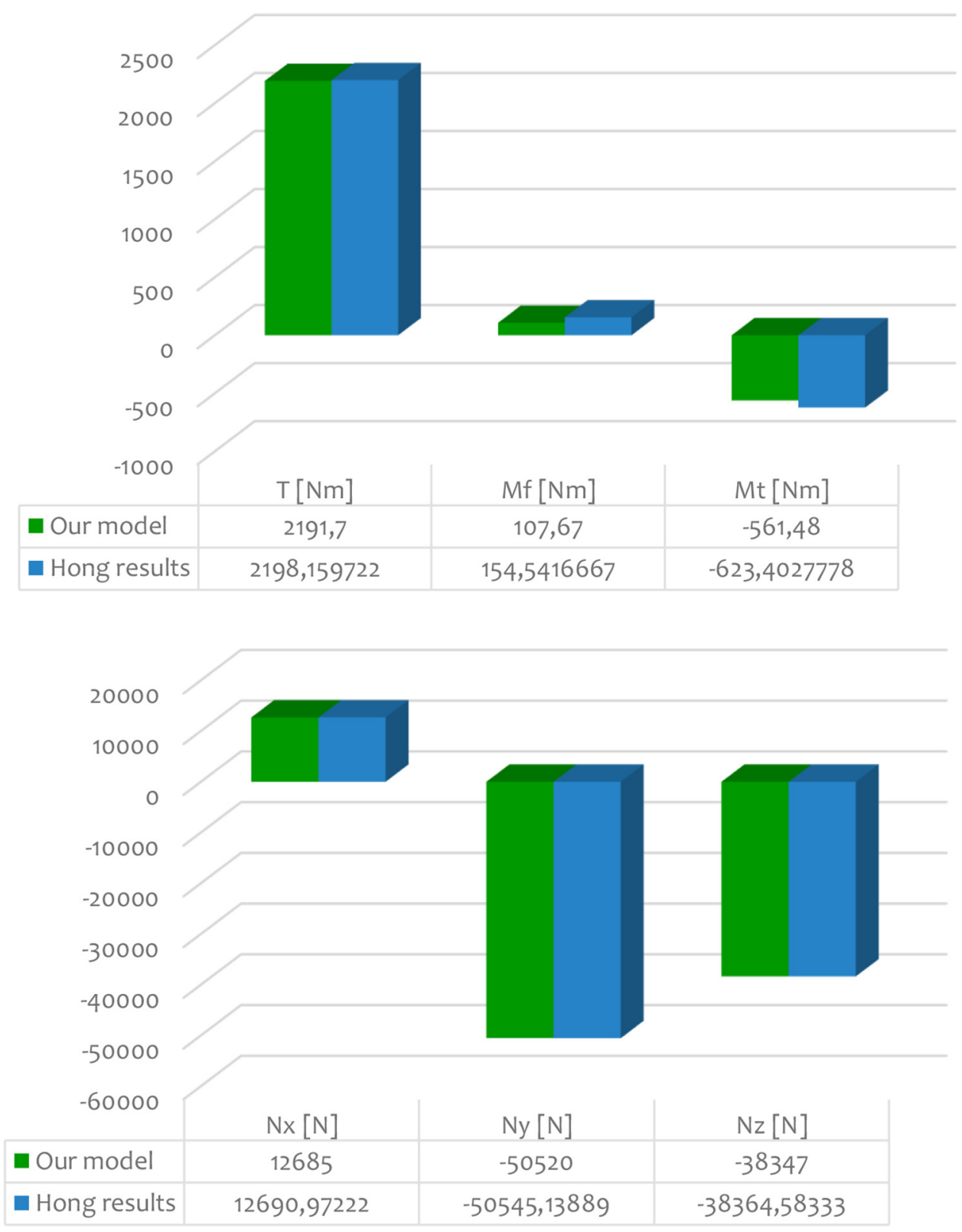

Fig. 17. Comparison between Hong results and our model.

approach, it has been shown that this type of approach makes it possible to have access, almost instantaneously, to the estimation of various key magnitudes of the operating conditions of the coupling. The lineic contact forces obtained from an approach mainly analytical are in very good agreement with one of the most refined numerical approaches for spline coupling without and with angular misalignment. From these lineic forces, an estimation of the contact peak pressures is done and can be used in wear model or fretting models. In the same way, one simply calculates from these lineic force distributions the reaction moments and the reaction forces.

The stiffness per unit length of the assembly as well as the tooth stiffness is also in agreement with the results of strictly numerical approaches. By integrating the stiffness

along the spline joint, a global stiffness for torsional vibration analysis is available.

For a given level of misalignment, the calculation of the bearing reactions (forces and moments) is easily accessible and thus makes it possible to estimate what the bearing dimensions should be. By knowing the stiffness of these bearings, the theoretical misalignment value can be corrected.

\section{Nomenclature}

$\theta$ $\psi$
Angle between teeth [rad]

Angular position on the hub/shaft [rad] Misalignment angle [rad] 


\begin{tabular}{|c|c|}
\hline$\alpha$ & Pressure angle [rad] \\
\hline$\rho$ & $\begin{array}{l}\text { Angle of embedment on the contact between } \\
\text { teeth [rad] }\end{array}$ \\
\hline $\overrightarrow{v_{C}}$ & Sliding speed on point $C[\mathrm{~m} / \mathrm{s}]$ \\
\hline$\omega$ & Rotation speed [rad/s] \\
\hline$K_{e}$ & $\begin{array}{l}\text { Elementary torsional stiffness per unit of } \\
\text { length }[\mathrm{N} / \mathrm{rad}]\end{array}$ \\
\hline$K, K_{t}$ & Torsional stiffness per unit of length $[\mathrm{N} / \mathrm{rad}]$ \\
\hline$K_{a}, K_{m}$ & $\begin{array}{l}\text { Torsional stiffness on the shaft and the hub } \\
\text { [Nm/rad] }\end{array}$ \\
\hline$u$ & Displacement $[\mathrm{m}]$ \\
\hline$x$ & Rotation axis of the spline coupling $[-]$ \\
\hline$y$ & Normal axis of the spline coupling [-] \\
\hline$z$ & Misalignment axis of the spline coupling $[-]$ \\
\hline$P$ & Force acting on teeth $[\mathrm{N}]$ \\
\hline$\mu P$ & Friction force $[\mathrm{N}]$ \\
\hline & Force applied according to the axis $k[\mathrm{~N}]$ \\
\hline$P_{a}^{n}, F_{n d}, F_{n q}$ & Lineic force on the indices $n$th tooth $[\mathrm{N} / \mathrm{m}]$ \\
\hline$\mu$ & Coulomb coefficient of friction $[-]$ \\
\hline$v$ & Poisson ratio $[-]$ \\
\hline$\chi$ & Kolosov constant $[-]$ \\
\hline G & Shear modulus $\left[\mathrm{N} / \mathrm{m}^{2}\right]$ \\
\hline$S$ & Sheared section $\left[\mathrm{m}^{2}\right]$ \\
\hline$I$ & Polar quadratic moment $\left[\mathrm{m}^{4}\right]$ \\
\hline$E$ & Young modulus $\left[\mathrm{N} / \mathrm{m}^{2}\right]$ \\
\hline$d, g$ & Index of the right $(\mathrm{d})$ and left $(\mathrm{g})$ sides $[-]$ \\
\hline$T$ & Torque around $x$ axis $[\mathrm{Nm}]$ \\
\hline$M_{F}$ & Bending moment around $y$ axis $[\mathrm{Nm}]$ \\
\hline$M_{T}$ & Bending moment around $z$ axis $[\mathrm{Nm}]$ \\
\hline$M_{f r, y}, M_{f r_{r} z}$ & Torques coming from friction [Nm] \\
\hline$M_{P, y}, M_{P, z}$ & Torques coming from bearing [Nm] \\
\hline & Teeth length $[\mathrm{m}]$ \\
\hline$b$ & Teeth width $[\mathrm{m}]$ \\
\hline$D, D_{P}$ & Diameter and primitive one $[\mathrm{m}]$ \\
\hline$R, R_{P}$ & Radius and primitive one $[\mathrm{m}]$ \\
\hline$\delta_{n}$ & Height of misalignment [m] \\
\hline$\delta_{p}$ & Parallel misalignment [m] \\
\hline$d_{d n}, d_{g n}$ & $\begin{array}{l}\text { Distance from application point } P \text { to axis } z \\
\text { [m] }\end{array}$ \\
\hline$a$ & $\begin{array}{l}\text { Half-length of contact on the curvilinear } \\
\text { abscissa }[\mathrm{m}]\end{array}$ \\
\hline$J$ & Clearance $[\mathrm{m}]$ \\
\hline int $_{n d}$, int $_{n g}$ & Interference depth $[\mathrm{m}]$ \\
\hline & Tooth index $[-]$ \\
\hline$n_{\max }$ & Total number of teeth $[-]$ \\
\hline$n_{c d}, n_{c g}$ & $\begin{array}{l}\text { Number of teeth in contact on the coupling } \\
{[-]}\end{array}$ \\
\hline$C_{n d}, C_{n g}$ & Contact condition $[-]$ \\
\hline
\end{tabular}

\section{References}

[1] F. Curà, A. Mura, Evaluation of the fretting wear damage on crowned splined couplings, in 2nd International Conference on Structural Integrity, ICSI 2017, 4-7 September 2017, Funchal, Madeira, Portugal

[2] S. Monti, M. Guagliano, Failure analysis of an involute spline coupling of an overhead bridge crane, Eng. Fail. Anal. 104, $321-330$ (2019)
[3] A. Mura, F. Curà, A. Di Molfetta, Investigation of bearings overloads due to misaligned splined shafts, Proc. Struct. Integr. 12, 52-57 (2018)

[4] J. Wang, J. Zhang, Z. Yao, R. Sun, Y. Zhao, Nonlinear characteristics of a multi-degree-of-freedom spur gear system with bending-torsional coupling vibration, Mech. Syst. Sig. Process. 121, 810-827 (2019)

[5] J. Hong, D. Talbot, A. Kahraman, Load distribution analysis of clearance-fit spline joints using finite elements, Mech. Mach. Theory 74, 42-57 (2014)

[6] R.A. Adey, J. Baynham, J.W. Taylor, Development of analysis tools for spline couplings, Proc. Inst. Mech. Eng. G 214, 347-357 (2000)

[7] M.A. Alfares, A.H. Falah, A.H. Elkholy, Clearance distribution of misaligned gear coupling teeth considering crowning and geometry variations, Mech. Mach. Theory 41, 1258-1272 (2006)

[8] F. Curà, A. Mura, Experimental and theoretical investigation about reaction moment in misaligned spline coupling, Mech. Syst. Signal Process. 45, 504-512 (2014)

[9] N. Fillot, Etude mécanique de l'usure, Thesis INSA Lyon, 2004

[10] J. Ding, W.S. Sum et al., Fretting fatigue predictions in a complex coupling. Int. J. Fatigue 29, 1229-1244 (2007)

[11] W. Qureshi, F. Curà, A. Mura, Characterization of fretting wear experiments on spline couplings by principal component analysis, Inst. Mech. Eng. Part J: J. Eng. Tribol. 231, 860-868 (2017)

[12] F. Curà, W. Qureshi, A. Mura, A methodological approach for incremental fretting wear formulation, Tribol. Lett. 64, 20 (2016)

[13] V. Cuffaro, F. Curà, A. Mura, Surface Characterization of spline coupling teeth subjected to fretting wear, Proc. Eng. 74, 135-142 (2014)

[14] J. Hong, D. Talbot, A. Kahraman, A semi-analytical load distribution model for side-fit involute splines, Mech. Mach. Theory 76, 39-55 (2014)

[15] S. Medina, A.V. Olver, An analysis of misaligned spline couplings, Proc. Inst. Mech. Eng. J 216, 269-278 (2002)

[16] S. Medina, A.V. Olver, Regimes of contact in spline coupling, J. Tribol. 124, 351-357 (2000)

[17] V. Hariharan, P. Srinivasan, Vibration analysis of parallel misaligned shaft with ball bearings system, S.J. Sci. Technol. 33, 61-68 (2011)

[18] M. Byrtus, V. Zeman, On modeling and vibration of gear drives influenced by nonlinear couplings, Mech. Mach. Theory 46, 375-397 (2011)

[19] A.H. Elkholy, M.A. Alfares, Misalignment loads in splined gear couplings, Int. J. Comput. Appl. Technol. 15, 128-137 (2002)

[20] J.R. Mancuso, Moments and forces imposed on power transmission systems due to misalignment of a crowned tooth coupling, Master's thesis, Pennsylvania State University, 1971

[21] Y. Guo, A. Lambert, R. Wallen, R. Errichello, J. Keller, Theoretical and experimental study on gear-coupling contact and loads considering misalignment, torque and friction influences, Mech. Mach. Theory 98, 242-262 (2016)

[22] P. Barrot, Analyse et modélisation du comportement des liaisons cannelées, Thesis INSA Toulouse, Novembre 2006

[23] A. Barrot, M. Paredes, M. Sartor, Extended equations of load distribution in the axial direction in a spline coupling, Eng. Fail. Anal. 16, 200-211 (2009) 
[24] A. Tjernberg, Load distribution in the axial direction in a spline coupling, Eng. Fail. Anal. 8, 357-570 (2001)

[25] J.-L. Ligier, M. Benoit, N. Pakpoor Gilani, Rapport interne COMATEC HEIG-VD, Mars 2018

[26] C. Dupertuis, Désalignement des liaisons cannelées et effets induits, Travail de Bachelor, HEIG-VD, septembre 2019
[27] A. Sackfield, C.E. Truman, D.A. Hills, The tilted punch under normal and shear load (with application to fretting tests), Int. J. Mech. Sci. 43, 1881-1892 (2001)

[28] R. Fargere, Simulation du comportement dynamique des transmissions par engrenages sur paliers hydrodynamiques, Thesis INSA Lyon, 2012

Cite this article as: C. Dupertuis, J.-L. Ligier, Contact pressure in misaligned spline couplings, Mechanics \& Industry 21, 510 $(2020)$ 